\title{
Measures of Autonomic Dysfunction in Diabetic and Idiopathic Gastroparesis
}

\author{
Mohammad Khalid Mohammad ${ }^{\mathrm{a}}$, Dominique J. Pepper ${ }^{\mathrm{b}}$, Archana Kedar ${ }^{\mathrm{a}}$, Feriyl Bhaijee, \\ Babajide Familoni ${ }^{\mathrm{d}}$, Hani Rashed ${ }^{\mathrm{e}}$, Teresa Cutts $^{\mathrm{f}}$, Thomas L. Abell ${ }^{\mathrm{a}, \mathrm{g}}$
}

\begin{abstract}
Background: Gastroparesis is a condition classically characterized by delayed gastric emptying and is associated with considerable morbidity. While the etiology of gastroparesis remains elusive, autonomic dysfunction may play an important role, especially as many patients with gastroparesis also have diabetes. The aim of this study was to determine whether measures of autonomic function differ between adults with diabetic gastroparesis (DG) and adults with idiopathic gastroparesis (IG).
\end{abstract}

Methods: Tests of systemic autonomic function were performed among 20 adults with GD (six men and 14 women, mean age: 42 years) and 21 adults with IG (seven men and 14 women, mean age: 37 years). Measures included vagal cholinergics by R-R interval percentage variation (RRI-PV) and sympathetic adrenergics by vasoconstriction to cold (VC) and postural adjustment ratio (PAR). The two groups were compared using Wilcoxon rank sum tests and linear regression analysis (STATA 10.0).

Results: In univariate analysis, the following autonomic measures differed significantly between $\mathrm{DG}$ and IG: VC $(\mathrm{P}=0.004)$, PAR (P $=0.045), \mathrm{VC}+\mathrm{PAR}(\mathrm{P}=0.002)$ and RRI-PV $(\mathrm{P}<0.001)$. In multivariate analysis $\left(\mathrm{P}=0.002, \mathrm{R}^{2}=0.55\right)$, only RRI-PV (adjusted odds ratio $(\mathrm{aOR}): 1.02,95 \%$ confidence interval $(\mathrm{CI}): 1.01$ - 1.03 ) differed significantly between DG and IG patients.

Manuscript accepted for publication August 15, 2016

aDivision of Gastroenterology, Hepatology \& Nutrition, Department of Medicine, University of Louisville, Louisville, KY, USA

${ }^{b}$ Department of Medicine, University of Mississippi Medical Center, Jackson, MS; Critical Care Medicine Department, National Institutes of Health, Bethesda, MD, USA

${ }^{\mathrm{c}}$ AmeriPath Indiana, Indianapolis, IN, USA

${ }^{d}$ US Army Research, Development and Engineering Command, NVESD, Ft. Belvoir, VA, USA

eNeuroscience Institute and Cancer Center, Methodist University Hospital, Memphis, TN, USA

fDivision of Public Health, Wake Forest School of Medicine, Winston Salem, NC, USA

gCorresponding Author: Thomas L. Abell, Division of Gastroenterology, Hepatology \& Nutrition, Department of Medicine, University of Louisville, 550 S. Jackson Street, ACB3 A3L15, Louisville, KY 40202, USA.

Email: thomas.abell@louisville.edu

doi: http://dx.doi.org/10.14740/gr713w
Conclusions: Vagal cholinergics are affected to a greater degree in DG compared to IG, suggesting that impaired vagal tone is not a universal mechanism for gastroparesis.

Keywords: Gastroparesis; Diabetes; Autonomic nervous system; Sympathetic function; Vagal dysfunction

\section{Introduction}

Gastroparesis is a cause of significant morbidity and diminished quality of life for more than 4 million Americans and almost $4 \%$ of the population, with a female/male ratio of $4: 1$ $[1,2]$. Although multiple conditions have been associated with gastroparesis, the majority of cases are idiopathic gastroparesis (IG) (etiology unknown) and diabetic gastroparesis (DG), each accounting for about one-third of all gastroparesis cases [3]. Patients with gastroparesis experience increased length of hospital stay and high total hospital costs, compared to patients with other gastric disorders including gastritis, gastroesophageal reflux disease, and gastric ulcer, resulting in a major economic burden to society [4]. Moreover, the incidence of hospitalizations due to gastroparesis increased 158\% from 1995 to 2004 .

Normal gastrointestinal motor function is comprised of a complex series of events that requires coordination of the sympathetic and parasympathetic nervous systems, neurons and pacemaker cells (called interstitial cells of Cajal) within the stomach and intestine, and the smooth muscle cells of the gut [2]. Abnormalities of this process are postulated to underlie the mechanisms of gastric stasis, namely gastroparesis. Its cardinal features are recurrent symptoms of upper gut motor dysfunction, nausea, vomiting, bloating, early satiety, and discomfort [5]. Weight loss, dehydration, electrolyte disturbances and malnutrition may develop in severe cases.

With the global rise in obesity-related diabetes, gastroparesis is being increasingly recognized in diabetes. In addition, diabetic autonomic neuropathy is a well-recognized entity $[6$, 7]. However, the role of autonomic dysfunction in gastroparesis is not clearly defined. Other factors operating in DG may include impaired neurotransmission, increased oxidative stress, loss of insulin-like growth factor-1 (IGF-1) as well as damage and loss of the interstitial cells of Cajal $[8,9]$. The pathogenesis of gastroparesis remains multifactorial and poorly understood, in part because of a lack of comprehensive mechanistic human studies. 
Table 1. Baseline Characteristics of Patients With Diabetic Gastroparesis (DG) and Patients With Idiopathic Gastroparesis (IG)

\begin{tabular}{llll} 
& DG $(\mathbf{n}=\mathbf{2 0})$ & IG $(\mathbf{n}=\mathbf{2 1})$ & P-value \\
\hline Female sex, n (\%) & $14(70 \%)$ & $14(67 \%)$ & 1.000 \\
Age in years, median (IQR) & $42(33-52)$ & $37(33-42)$ & 0.108 \\
Race (white), n (\%) & $17(85 \%)$ & $18(86 \%)$ & 1.000 \\
Symptoms duration in months, median (IQR) & $15(12-24)$ & $33(15-60)$ & 0.030 \\
Percentage vasoconstriction, median (IQR) & $45(26-84)$ & $84(69-98)$ & 0.005 \\
Postural adjustment ratio (PAR), median (IQR) & $16(11-27)$ & $27(15-44)$ & 0.046 \\
Vasoconstriction and PAR, median (IQR) & $57(39-106)$ & $111(92-127)$ & 0.002 \\
R-R interval percentage variation, median (IQR) & $8(5-15)$ & $33(23-51)$ & $<0.001$ \\
\hline
\end{tabular}

$P<0.05$ considered significant. IQR: interquartile range.

The purpose of the current study was firstly to determine if autonomic dysfunction plays an etiologic role in the development of two major forms of gastroparesis, DG and IG, and secondly to investigate whether measures of autonomic function differed between patients with DG and patients with IG.

\section{Patients and Methods}

\section{Patients}

Patients with symptoms of DG and IG were recruited from the University of Tennessee, Memphis. No patients had previous gastric surgery. The Institutional Review Board of the University of Tennessee, Memphis, approved the study.

We defined patients with gastroparesis as having prolonged, recurrent symptoms of upper gut motor dysfunction, such as nausea, vomiting, and abdominal pain, with delayed gastric emptying, and absence of structural abnormalities of the gastrointestinal tract. Diabetic patients with gastroparesis were regarded as having DG. Patients with gastroparesis who did not have diabetes, a history of gastric surgery or an identifiable cause of gastroparesis were regarded as having IG.

\section{Methods}

For patients meeting criteria for DG or $\mathrm{IG}$, we recorded age, sex (male/female), patient-reported race (Caucasian/AfricanAmerican) and duration of symptoms prior to initial assessment. We assessed autonomic function in all patients during daylight hours using two measures of adrenergic function, and one measure of cholinergic function. Hathaway et al. have described these tests in detail [10].

\section{Sympathetic adrenergic assessment}

We assessed sympathetic adrenergic function by calculating the postural adjustment ratio (PAR) and the percentage of reflex vasoconstriction to cold $(\mathrm{VC} \%)$. The PAR and the
$\mathrm{VC} \%$ were obtained by measuring changes in the peripheral circulation using capillary photoplethysmography. The PAR was determined while the patient was sitting. The patient's left hand and arm rested at a $45^{\circ}$ angle above heart level on a comfortable chair armrest for about $5 \mathrm{~min}$. After recording stable peripheral blood flow, the patient was asked to drop the arm down freely, next to the side of their chair, without touching or holding anything for $1 \mathrm{~min}$. The change in blood flow was then calculated and reported as the PAR. Next, the patient's hand was immersed in cold water $\left(14^{\circ} \mathrm{C}\right)$ for $1 \mathrm{~min}$, and blood flow was measured in the opposite hand. The percentage change in blood flow from baseline was reported as the $\mathrm{VC} \%$.

\section{Vagal cholinergic assessment}

Vagal cholinergic measures were determined using the R-R interval percentage variation (RRI-PV) as elicited by respiratory maneuvers. This was determined by measuring varying heart rates during respiration. The patient was placed in a supine position, with instructions to breathe deeply in-and-out for about six cycles per minute. During deep inhalation, the maximum heart rate was recorded; during deep expiration, the lowest heart rate was recorded. The percentage change in heart rate with respiration (RRI-PV) was calculated by subtracting heart rate during inspiration from the heart rate during expiration, divided by heart rate with expiration (RRI-PV $=(\mathrm{RRI}$ expiration - RRI inspiration)/RRI expiration) $\times 100$.

\section{Statistical analysis}

We analyzed data using statistical software Stata 10.0 (Texas, USA) and Graphpad Prism 5.0b. Most variables were right (non-normal) skewed but had normal distributions with logarithmic transformation (Shapiro-Wilk test) (Supplementary Table 1, www.gastrores.org); we described these variables using medians and interquartile ranges (IQRs). Proportions were calculated for categorical variables and described using 95\% confidence intervals (CIs). We used Fisher's exact test to determine which categorical variables differed significantly between patients with DG and IG. Similarly, we used the Wilcoxon rank 
Table 2. Linear Regression Model of Variables Associated With Diabetic Gastroparesis, Compared With Idiopathic Gastroparesis

\begin{tabular}{llll} 
& P-value & aOR & 95\% CI \\
\hline Age & 0.923 & NS & - \\
Percentage vasoconstriction (VC\%) & 0.736 & NS & - \\
Postural adjustment ratio (PAR) & 0.742 & NS & - \\
VC\% and PAR & 0.83 & NS & - \\
R-R interval percentage variation & 0.001 & 1.02 & $1.01-1.03$ \\
Symptoms duration & 0.597 & NS & - \\
\hline
\end{tabular}

$P<0.05$ considered significant. Linear regression model: $P=0.002, R^{2}=0.548$.

sum tests to determine which continuous variables differed significantly between patients with DG and IG. Variables with a $\mathrm{P}$-value $<0.2$ were incorporated into our linear regression analysis to explore the relationships of these variables across the two forms of gastroparesis. A backward stepwise linear model was proposed to quantify these relationships; this model was reported using adjusted odds ratios (aORs) and 95\% CIs. We fitted the model using the likelihood ratio, which was logarithmically transformed to generate the Chi-squared statistic.

\section{Results}

\section{Baseline characteristics}

We enrolled 20 patients with DG and 21 patients with IG (Table 1). Most participants were Caucasian (DG: 17/20; IG: 18/21), female (DG: 14/20; IG: 14/21), and middle-aged (DG median age: 42 years (IQR: 33 - 52); IG median age: 37 years (IQR: 33 - 42)). The median duration of symptoms prior to presentation was greater in patients with IG (median 33 months, IQR: 15 - 60) compared to patients with DG (median 15 months, IQR: 12 - 24, $\mathrm{P}=0.03$ ).

\section{Measures of autonomic function}

In univariate analysis, all four measures of autonomic function differed significantly between DG and IG (Table 1). These measures included VC $(\mathrm{P}=0.005)$, PAR $(\mathrm{P}=0.046), \mathrm{VC}+$ PAR $(\mathrm{P}=0.002)$, and RRI-PV $(\mathrm{P}<0.001)$. The following variables were entered into a linear regression model: VC, PAR, $\mathrm{VC}+\mathrm{PAR}$, RR-IPV, age, and duration of symptoms $(\mathrm{P}<0.2)$. Using our linear regression model $\left(P=0.002, R^{2}=0.548\right)$, only RRI-PV differed significantly between patients with DG and IG (aOR: 1.02, 95\% CI: $1.01-1.03, \mathrm{P}=0.001$ ) (Table 2). The median RRI-PV among patients with DG was 8 (IQR: 5 -

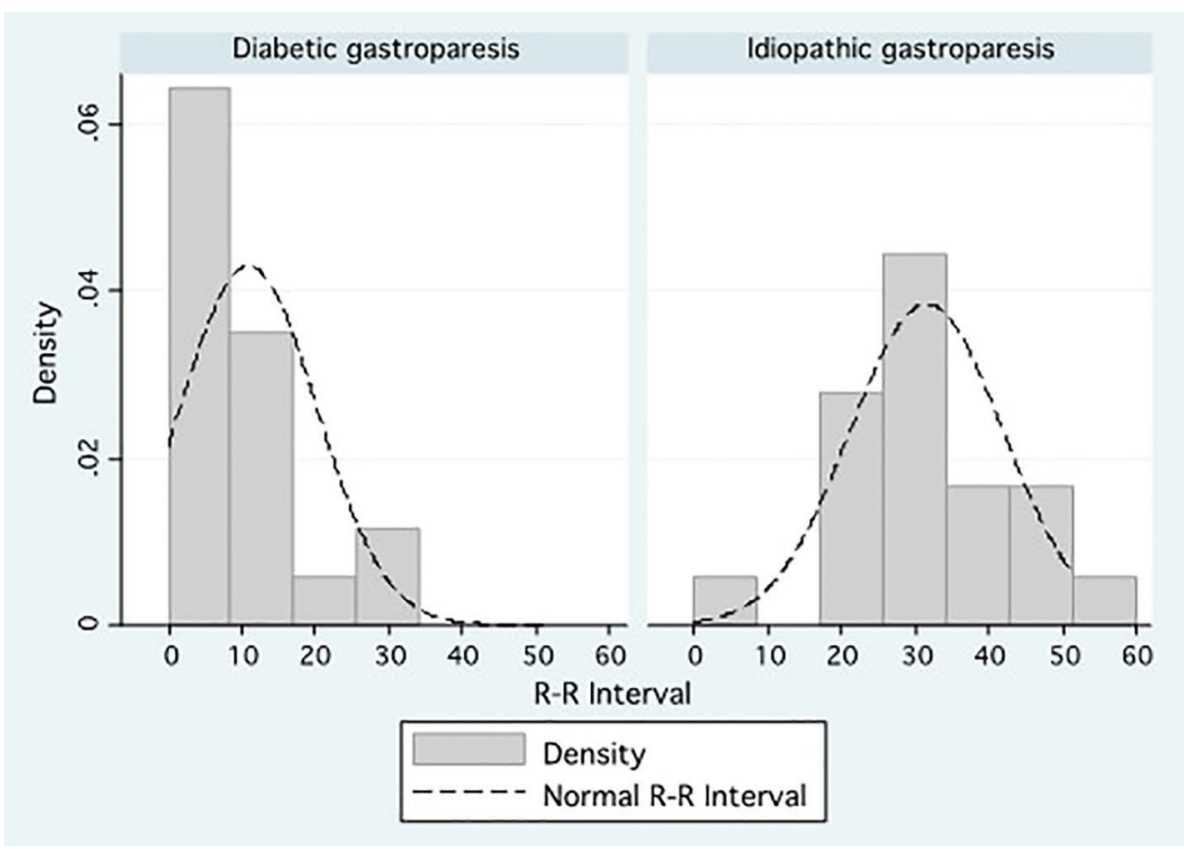

Figure 1. Density distributions comparing the percentage R-R interval variation between patients with diabetic gastroparesis and patients with idiopathic gastroparesis. 
15), compared to the median RRI-PV among patients with IG, which was 33 (IQR: 23 - 51) (Fig. 1).

\section{Discussion}

The etiopathogenesis of gastroparesis is largely unknown, but has been attributed to various factors, such as disorders of autonomic function and neurotransmission, increased oxidative stress, loss of IGF-1, as well as damage and loss of the interstitial cells of Cajal $[8,9]$. In this study, we explored autonomic measures among gastroparesis and found that vagal cholinergics were dysfunctional in patients with DG, but not in those with IG. Our finding suggests that abnormal vagal tone is not a common mechanism for gastroparesis.

In this study, we assessed patients with DG and IG, the two largest subgroups of patients that comprise gastroparesis. These patients were meticulously assessed and characterized, and alternate causes of gastroparesis were excluded. Patients with post-surgical gastroparesis (with vagotomy or damage to the vagus nerve) were not included in our study. It is logical to conclude that measures of vagal cholinergic function would be impaired in patients with post-surgical gastroparesis.

We observed that RRI-PV among DG and IG was 8 and 33, respectively. Measures of cardiovascular autonomic neuropathies were utilized as surrogates for markers of gastrointestinal autonomic neuropathies in our study. Cardiac autonomic neuropathy, gastric autonomic neuropathy and peripheral neuropathy are closely related, and R-R interval variation during deep breathing is a good indicator of diabetic gastropathy [11]. Cardiovascular autonomic neuropathies are the most clinically important form of diabetic autonomic neuropathy [7] and are typically measured by RRI-PV, PARs and the VC\%.

Our finding that RRI-PV was impaired in DG may explain why a prior randomized controlled trial showed gastroesophageal stimulators were almost exclusively of benefit to patients with DG rather than those with IG [12]. Gastric mucosal nerves from diabetic subjects typically have reduced density and abnormal morphology compared to control subjects [13]. Moreover, histologic abnormalities in patients with DG and IG are heterogeneous and include myenteric inflammation, decreased innervation, and reduction of interstitial cells of Cajal [14].

Of interest is the preponderance of women with gastroparesis in our study. In two large investigations, over $80 \%$ of patients with gastroparesis were female $[12,15]$. This female preponderance is generally attributed to women having delayed gastric emptying compared to men [16], which may be mediated by differences in ovarian hormones [17] or altered enteric neural conduction and propulsion. Complex interactions exist between the enteric, autonomic and central nervous systems. Future studies may better characterize the exact nature of these interactions, and their relationship to the pathogenesis of gastroparesis. Moreover, besides post-viral illnesses [18, 19] and gastroesophageal reflux, the etiology of IG is largely unknown. Further studies may determine the etiopathogenesis of gastroparesis in terms of differences in autonomic function between DG and IG patients.

The strength of this study is to better quantify the auto- nomic differences between diabetic patients and those without diabetes. The limitation of the current study is the relatively small sample number; large-scale studies are still needed.

\section{Conclusion}

Vagal cholinergics are affected to a greater degree in DG, compared to IG, suggesting that vagal tone is not a universal mechanism for gastroparesis.

\section{Acknowledgments}

The authors would like to thank the staff of the autonomic function laboratory at the University of Tennessee, Memphis. The authors would also like to thank the staff of the GI Division at the University of Mississippi, where some of the authors previously worked when analyzing the data. Lastly, the authors would like to thank Catherine McBride of the University of Louisville, Division of Gastroenterology, Hepatology and $\mathrm{Nu}-$ trition for help with manuscript preparation and submission.

\section{Source of Funding}

Internal funding.

\section{Disclosures/Conflicts of Interest}

None.

\section{Author Contributions}

Analysis and interpretation of data: MKM, DJP, AK, HR, TC, and TA; drafting of the manuscript: MKM, DJP, AK, FB, BF, $\mathrm{TC}$, and TA; critical revision of the manuscript: MKM, AK, FB, BF, HR, TC, and TA; statistical analysis: MKM, DJP, TC, and TA; study concept and design: TC; study concept and design: TA.

\section{References}

1. Abell TL, Bernstein RK, Cutts T, Farrugia G, Forster J, Hasler WL, McCallum RW, et al. Treatment of gastroparesis: a multidisciplinary clinical review. Neurogastroenterol Motil. 2006;18(4):263-283.

2. Grover M, Farrugia G, Lurken MS, Bernard CE, Faussone-Pellegrini MS, Smyrk TC, Parkman HP, et al. Cellular changes in diabetic and idiopathic gastroparesis. Gastroenterology. 2011;140(5):1575-1585 e1578.

3. Vittal H, Farrugia G, Gomez G, Pasricha PJ. Mechanisms of disease: the pathological basis of gastroparesis - a review of experimental and clinical studies. Nat Clin Pract Gastroenterol Hepatol. 2007;4(6):336-346. 
4. Tang DM, Friedenberg FK. Gastroparesis: approach, diagnostic evaluation, and management. Dis Mon. 2011;57(2):74-101.

5. Camilleri M, Parkman HP, Shafi MA, Abell TL, Gerson L. Clinical guideline: management of gastroparesis. Am J Gastroenterol. 2013;108(1):18-37; quiz 38.

6. Neshatian L, Gibbons SJ, Farrugia G. Macrophages in diabetic gastroparesis - the missing link? Neurogastroenterol Motil. 2015;27(1):7-18.

7. Boulton AJ, Vinik AI, Arezzo JC, Bril V, Feldman EL, Freeman R, Malik RA, et al. Diabetic neuropathies: a statement by the American Diabetes Association. Diabetes Care. 2005;28(4):956-962.

8. Hasler WL. Management of gastroparesis. Expert Rev Gastroenterol Hepatol. 2008;2(3):411-423.

9. Patrick A, Epstein O. Review article: gastroparesis. Aliment Pharmacol Ther. 2008;27(9):724-740.

10. Hathaway DK, Abell T, Cardoso S, Hartwig MS, el Gebely S, Gaber AO. Improvement in autonomic and gastric function following pancreas-kidney versus kidney-alone transplantation and the correlation with quality of life. Transplantation. 1994;57(6):816-822.

11. Asakawa H, Onishi M, Hayashi I, Fukuda A, Tokunaga $\mathrm{K}$. Comparison between coefficient of R-R interval variation and gastric emptying in type 2 diabetes mellitus patients. J Gastroenterol Hepatol. 2005;20(9):1358-1364.

12. Abell T, McCallum R, Hocking M, Koch K, Abrahamsson H, Leblanc I, Lindberg G, et al. Gastric electrical stimulation for medically refractory gastroparesis. Gastroenterol- ogy. 2003;125(2):421-428.

13. Selim MM, Wendelschafer-Crabb G, Redmon JB, Khoruts A, Hodges JS, Koch K, Walk D, et al. Gastric mucosal nerve density: a biomarker for diabetic autonomic neuropathy? Neurology. 2010;75(11):973-981.

14. Harberson J, Thomas RM, Harbison SP, Parkman HP. Gastric neuromuscular pathology in gastroparesis: analysis of full-thickness antral biopsies. Dig Dis Sci. 2010;55(2):359-370.

15. Parkman HP, Yates K, Hasler WL, Nguyen L, Pasricha PJ, Snape WJ, Farrugia G, et al. Clinical features of idiopathic gastroparesis vary with sex, body mass, symptom onset, delay in gastric emptying, and gastroparesis severity. Gastroenterology. 2011;140(1):101-115.

16. Sfarti C, Trifan A, Hutanasu C, Cojocariu C, Singeap AM, Stanciu C. Prevalence of gastroparesis in type 1 diabetes mellitus and its relationship to dyspeptic symptoms. J Gastrointestin Liver Dis. 2010;19(3):279-284.

17. Datz FL, Christian PE, Moore J. Gender-related differences in gastric emptying. J Nucl Med. 1987;28(7):12041207.

18. Oh JJ, Kim CH. Gastroparesis after a presumed viral illness: clinical and laboratory features and natural history. Mayo Clin Proc. 1990;65(5):636-642.

19. Soykan I, Sivri B, Sarosiek I, Kiernan B, McCallum RW. Demography, clinical characteristics, psychological and abuse profiles, treatment, and long-term follow-up of patients with gastroparesis. Dig Dis Sci. 1998;43(11):23982404. 